\section{Avaliação de efetividade da atenção domiciliar de uma cooperativa médica de Belo Horizonte, Minas Gerais, Brasil}

\author{
Home care effectiveness assessment in a health \\ maintenance organization in Belo Horizonte, \\ Minas Gerais State, Brazil
}

\section{Evaluación de la eficacia de un programa de atención domiciliaria en una cooperativa médica de Belo Horizonte, Minas Gerais, Brasil}

Fernando Martín Biscione 1

Daniele Araújo Campos Szuster 1 Eliane de Freitas Drumond 1,2 Graziele Umbelina Alves Ferreira ${ }^{1}$ Maria Aparecida Turci 3 Jorge Faria Lima Júnior 1 Sérgio Adriano Loureiro Bersan 4

\begin{abstract}
A retrospective cohort study was performed to assess the impact of a Case Management Home Care Program supplied by the Unimed-BH medical cooperative on hospitalization-free survival time among eligible patients 60 years or older. A Cox proportional hazards model was fitted to assess the impact of home visits by health professionals on hospitalization-free survival time in a sample of 2,943 elders, while adjusting for patient age, physical dependence, medicines, feeding route, pressure ulcers, supplemental oxygen therapy, cognitive impairment, outpatient visits, and hospitalizations in the preceding quarter. Risk factors for shorter hospitalization-free survival time were: degree of physical dependence, enteral nutrition, supplemental oxygen therapy, pressure ulcers, and hospital admissions in the previous quarter. Higher rates of home visits by physicians and nurses showed a protective doseresponse effect on hospitalization-free survival time. The data suggest that regular home visits by physicians and nurses lengthen hospitalization-free survival time among elderly patients enrolled in the program.
\end{abstract}

Home Nursing; Aged; Health Technology Evaluation

\section{Resumo}

Foi realizado estudo de coorte retrospectiva com o objetivo de avaliar o impacto do plano de cuidados do Programa de Atenção Domiciliar da Unimed-BH, modalidade Gerenciamento de Casos (PrGC/AD), sobre o tempo livre de hospitalização entre os pacientes com 60 anos ou mais assistidos pelo programa. Utilizou-se o modelo de Cox para avaliar o efeito do intervalo entre as visitas domiciliares dos profissionais do programa sobre o tempo livre de hospitalização de 2.943 idosos, ajustado por idade, medicamentos em uso, via de alimentação, úlcera de pressão, déficit cognitivo, dependência física, oxigenioterapia, consultas ambulatoriais e hospitalizações no trimestre anterior. Foram fatores de risco para menor tempo livre de hospitalização: o grau de dependência física, alimentação enteral, oxigenioterapia suplementar, úlceras de pressão e hospitalizações no trimestre anterior. Observouse efeito protetor dose-resposta da frequência de visitas médicas e de enfermagem. Os resultados sugerem que visitas domiciliares regulares de médico e enfermeiro aumentam significativamente o tempo livre de hospitalização nos pacientes assistidos pelo PrGC/AD.

Assistência Domiciliar; Idoso; Avaliação de Tecnologias de Saúde 


\section{Introdução}

Nas últimas décadas, o rápido crescimento da população idosa brasileira veio acompanhado por significativo aumento da prevalência de doenças cronicodegenerativas e de incapacidade funcional, geradores de demanda por todos os níveis dos serviços de saúde e de elevado ônus social 1,2,3. Doenças crônicas e incapacitantes, de alta prevalência na população idosa, aliadas ao modelo de atenção em curso, ainda fortemente centrado no hospital, favorecem o atendimento da pessoa idosa em serviços de urgência/emergência ou durante a internação no hospital, em detrimento da atenção ambulatorial. A estratégia pública de saúde, no entanto, tem favorecido o acesso à atenção ambulatorial dos idosos, com consequente redução das hospitalizações ${ }^{4}$. Estratégia de especial importância se considerada a significativa diminuição da capacidade funcional e da qualidade de vida subsequente às internações nessa população ${ }^{5}$.

Programas de atenção domiciliar se apresentam como alternativas de atenção à saúde do idoso frágil 6 . Amplamente utilizados em países desenvolvidos, passaram a expandir sua atuação no Brasil somente a partir da última década do século XX, tanto no setor público, quanto no privado 7 . Entretanto, sua efetividade ainda não foi adequadamente avaliada em nosso país, especialmente no que se refere à população idosa.

Avaliações da efetividade de programas de atenção à saúde são um desafio a ser enfrentado em cenários dominados pela incorporação desmesurada de novas tecnologias, elevação de custos assistenciais e recursos limitados. Este estudo teve como objetivo principal avaliar o impacto do plano de cuidados executado pela equipe de atenção domiciliar da Cooperativa de Trabalho Médico Unimed Belo Horizonte (Unimed-BH) no tempo livre de internação hospitalar, entre os idosos assistidos pelo programa em 2011.

\section{Material e métodos}

Foi conduzido estudo de coorte retrospectiva, utilizando-se das informações da população idosa (60 anos ou mais) atendida no segundo e terceiro trimestres de 2011 pelo Programa de Atenção Domiciliar da Unimed-BH, na sua modalidade Gerenciamento de Casos (PrGC/AD).

O PrGC/AD tem como principal proposta a vigilância em saúde do paciente no domicílio, por meio do apoio ao doente, à sua família e aos seus cuidadores na manutenção do estado de saúde, bem como na prevenção de doenças e sequelas. São elegíveis para o PrGC/AD pacientes com di- ficuldade ou impossibilidade de locomoção, com comprometimento variável na realização de atividades da vida diária, portadores de polipatologias crônicas e quadros demenciais. A maior parte da casuística é formada por pacientes com doenças neurodegenerativas (incluindo demência) ou sequelas de acidente vascular encefálico. Não são elegíveis para o programa pacientes que estejam em uso de ventilação mecânica ou em cuidados paliativos. Os pacientes admitidos devem contar com apoio de cuidador, e a adesão ao programa é voluntária. A participação, contudo, está sujeita à verificação dos critérios de elegibilidade pela equipe de auditoria de enfermagem domiciliar.

A solicitação de admissão no PrGC/AD pode ser feita pelo médico assistente ou pelo próprio paciente ou familiar. Então, a auditoria de enfermagem domiciliar faz uma avaliação do paciente, utilizando ficha padronizada para classificação da complexidade clínica. A classificação baseia-se nos seguintes itens 8: faixa etária; estado cognitivo conforme versão em português da escala Clinical Dementia Rating; grau de dependência para atividades básicas da vida diária (escala de Katz) 9; via de entrada de ar; número de medicamentos em uso; via de alimentação; úlcera de pressão. Cada item da avaliação produz um resultado numérico, e a soma dos itens gera um escore de risco que permite classificar o paciente como sendo de baixa, média ou alta complexidade, determinando seu plano de cuidados. Detalhes sobre essa classificação podem ser consultados em Valle et al. ${ }^{8}$.

As equipes do PrGC/AD são compostas por médico, enfermeiro, fisioterapeuta, fonoaudiólogo, psicólogo e nutricionista, os quais atuam de acordo com o plano de cuidados estabelecido. A frequência e a categoria dos profissionais (médico, enfermeiro etc.) que realizaram as visitas domiciliares foram aferidas mediante auditorias domiciliares trimestrais conduzidas por uma equipe de enfermagem. Os dados apresentados neste estudo se referem às auditorias conduzidas no segundo e terceiro trimestres de 2011; assim, cada paciente recebeu no mínimo uma e no máximo três auditorias domiciliares, determinando o tempo de observação de cada paciente do estudo. Tanto esta avaliação quanto a avaliação da complexidade dos pacientes no momento da admissão no PrGC/AD foram realizadas nas condições habituais do serviço, não havendo, portanto, qualquer controle das variáveis do estudo ou interferência na assistência prestada.

Modelo de riscos proporcionais de Cox foi utilizado para avaliar o impacto do intervalo entre as visitas domiciliares dos profissionais de saúde sobre o tempo decorrido até a primeira 
internação, a partir da admissão do paciente no PrGC/AD. Pacientes que não foram hospitalizados no período de observação foram censurados na data da última auditoria domiciliar. $\mathrm{O}$ modelo considerou, como variáveis de ajuste, a frequência de consultas médicas ambulatoriais com especialidades básicas durante a permanência do paciente no PrGC/AD e o número de internações no trimestre anterior à admissão; considerou, também, as variáveis de complexidade clínica conforme ficha de avaliação da auditoria descrita anteriormente, que permitiram aferir o risco basal do paciente. $\mathrm{O}$ modelo final foi construído por eliminação manual de variáveis passo a passo, valendo-se do modelo com todas as variáveis candidatas. O nível de significância estipulado para permitir a permanência das variáveis explicativas no modelo final foi de 0,05. Utilizou-se o pacote Stata versão 12.1 (Stata Corp., College Station, Estados Unidos) para análise estatística.

Foi preservada a confidencialidade e o sigilo das informações dos sujeitos envolvidos no estudo, individual ou coletivamente considerados. Os preceitos éticos da pesquisa que envolve seres humanos, presentes na Resolução no 196/96 do Conselho Nacional de Saúde e na Declaração de Helsinki, foram observados.

\section{Resultados}

A população do estudo foi composta por 2.943 idosos assistidos pelo PrGC/AD em 2011. A idade média foi de 85,2 anos, e $74 \%$ eram mulheres. Quase $90 \%$ dos pacientes eram de alta ou média complexidade (Tabela 1). Entre aqueles de alta complexidade, foi maior a proporção de idosos com 80 anos ou mais, com dependência total para atividades básicas da vida diária, com demência estabelecida e com uso de alimentação por via enteral. Entre os de baixa complexidade, foi mais frequente a dependência parcial para atividades básicas da vida diária e o uso de mais de cinco medicamentos ao dia.

Foram internados durante o acompanhamento 697 pacientes (24\%). Para os que não tiveram qualquer internação durante o acompanhamento, censurados na data da última auditoria domiciliar, a mediana de tempo de acompanhamento foi de 187 dias (média de 167 dias). Para aqueles que tiveram pelo menos uma internação durante o acompanhamento, a mediana de tempo até a primeira ocorrência foi de 65 dias (média de 79 dias). As causas mais frequentes foram pneumonia (26\%); infecção do trato urinário (8\%); insuficiência respiratória (5\%); acidente vascular encefálico (4\%); insuficiência cardíaca
(4\%); fratura de fêmur (3\%); hemorragia digestiva (3\%); e depleção de volume (3\%). Alta por óbito foi registrada em 27 (1\%) pacientes internados.

Na Tabela 2, o modelo de Cox mostra o impacto de todas as variáveis explicativas analisadas sobre o tempo livre de internação hospitalar dos pacientes admitidos no PrGC/AD. Nesse modelo, o maior grau de dependência para atividades básicas da vida diária, a alimentação pela via enteral, a oxigenioterapia suplementar, a presença de úlcera de pressão e o antecedente de internação hospitalar no trimestre anterior à admissão no PrGC/AD foram fatores de risco para menor tempo livre de internação hospitalar após a entrada no programa. $\mathrm{O}$ intervalo entre as visitas domiciliares por médico ou enfermeiro apresentou associação, do tipo dose-resposta, com o tempo livre de internação hospitalar: quanto menor o intervalo entre as visitas, maior o tempo livre de internação. Essa associação manifestou-se em razões de risco de internação significativamente inferiores à unidade, quando comparados pacientes que receberam visitas em intervalos frequentes (menor que ou igual a um mês, ou menor que ou igual a dois meses) aos que receberam visitas mais esparsas (maior que dois meses). Foi observado também efeito protetor das visitas do nutricionista, do fisioterapeuta e das consultas médicas ambulatoriais com especialidades básicas.

\section{Discussão}

Os resultados obtidos sugerem que a frequência de visitas domiciliares dos profissionais de saúde do PrGC/AD impacta significativamente no tempo livre de internação hospitalar dos pacientes assistidos pelo programa. Espera-se, com isso, consequente redução dos efeitos deletérios das hospitalizações, especialmente no que se refere aos cuidados pessoais, mobilidade e locomoção ${ }^{9}$, aspecto prioritário da Política Nacional de Saúde da Pessoa Idosa 10. À exceção do grau de déficit cognitivo, as variáveis que compõem a ficha de classificação de complexidade clínica avaliada neste estudo mostraram-se preditoras do risco de internação dos idosos. Ressalte-se a alta proporção de pacientes da nossa coorte com importante comprometimento de seu estado geral e elevado grau de dependência. A preponderância de mulheres pode ser reflexo do crescimento desigual da expectativa de vida entre os sexos, mais significativo nas mulheres do que nos homens. Esse fenômeno foi relatado no Brasil por Veras 11 há mais de duas décadas, e pode ser atribuído a fatores biológicos e/ou exposição desigual a fatores de risco à saúde. 
Tabela 1

Características da população de estudo, de acordo com o nível de complexidade.

\begin{tabular}{|c|c|c|c|c|c|c|}
\hline \multirow[t]{2}{*}{ Característica } & \multicolumn{2}{|c|}{ Baixa complexidade } & \multicolumn{2}{|c|}{ Média complexidade } & \multicolumn{2}{|c|}{ Alta complexidade } \\
\hline & $n(N=353)$ & $\%(12)$ & $n(N=1.376)$ & $\%(47)$ & $n(N=1.214)$ & $\%(41)$ \\
\hline Feminino & 251 & 71 & 994 & 72 & 942 & 78 \\
\hline Masculino & 102 & 29 & 382 & 28 & 272 & 22 \\
\hline \multicolumn{7}{|l|}{ Faixa etária (anos) } \\
\hline $60-69$ & 37 & 10 & 93 & 7 & 38 & 3 \\
\hline $70-79$ & 99 & 28 & 295 & 21 & 91 & 7 \\
\hline 80 ou mais & 217 & 61 & 988 & 72 & 1.085 & 89 \\
\hline \multicolumn{7}{|c|}{ Grau de dependência para atividades básicas da vida diária * } \\
\hline Independente & 94 & 29 & 47 & 3 & 17 & 1 \\
\hline Parcial & 216 & 67 & 988 & 73 & 549 & 45 \\
\hline \multicolumn{7}{|l|}{ Via de alimentação * } \\
\hline Oral & 320 & 100 & 1.330 & 98 & 873 & 72 \\
\hline Enteral & 1 & 0 & 24 & 2 & 335 & 28 \\
\hline \multicolumn{7}{|l|}{ Cognição * } \\
\hline Sem déficit & 236 & 74 & 319 & 24 & 131 & 11 \\
\hline Déficit leve & 76 & 24 & 650 & 48 & 393 & 33 \\
\hline Demência & 9 & 3 & 385 & $28 \%$ & 684 & 57 \\
\hline \multicolumn{7}{|c|}{ Número de medicamentos em uso * } \\
\hline Sem uso & 3 & 1 & 19 & 1 & 14 & 1 \\
\hline $1-5$ & 106 & 33 & 538 & 40 & 510 & 42 \\
\hline Mais de 5 & 212 & 66 & 797 & 59 & 684 & 57 \\
\hline \multicolumn{7}{|c|}{ Via de entrada de ar * } \\
\hline Ar ambiente & 316 & 98 & 1.302 & 96 & 1.081 & 89 \\
\hline Traqueostomia & 0 & 0 & 6 & 0 & 12 & 1 \\
\hline \multicolumn{7}{|l|}{ Úlcera de pressão * } \\
\hline Pele íntegra & 299 & 93 & 1.227 & 91 & 981 & 81 \\
\hline Grau I ou II & 18 & 6 & 118 & 9 & 186 & 15 \\
\hline Grau III ou IV & 4 & 1 & 9 & 1 & 41 & 3 \\
\hline
\end{tabular}

* Dados ausentes para 32, 22 e 6 pacientes de baixa, média e alta complexidade, respectivamente.

Avaliações da efetividade da atenção domiciliar são escassas e têm mostrado resultados discordantes. Alguns estudos observaram benefícios relacionados à melhora na qualidade de vida, redução da mortalidade e admissão em instituições de longa permanência; os efeitos sobre o risco de internação hospitalar, porém, são incertos 12,13,14. Talvez, em virtude das dificuldades inerentes à avaliação dos programas de atenção domiciliar, não foram encontradas, pelos autores, publicações brasileiras comparáveis com o presente estudo. As avaliações de tecnologias direcionadas à atenção ao idoso, ainda incipientes no Brasil, precisam ser ampliadas, pois podem apontar para melhores soluções clínicas e econô- micas na assistência dessa população, especialmente em situações polêmicas, como as ligadas à efetividade da atenção domiciliar.

Discutindo sobre os pontos de fragilidade e a multiplicidade de conflitos envolvidos na atenção domiciliar ao idoso, Floriani \& Schramm 12 ressaltam a necessidade de se evitar a sobrecarga de atividades dos cuidadores domiciliares e de se refletir sobre as possíveis consequências da qualificação destes na saúde dos idosos. Propõem considerar, também, a forma de organização dos programas de atenção domiciliar, a dinâmica das relações familiares, além da capacidade de construção de relações positivas entre os profissionais de saúde e a família ${ }^{12}$. Assim, as 
Modelo de riscos proporcionais de Cox para tempo livre de internação hospitalar.

\begin{tabular}{|c|c|c|c|}
\hline Variável & Razão de riscos & IC95\% & Valor de $p$ \\
\hline \multicolumn{4}{|l|}{ Intervalo entre as visitas médicas (meses) [referência: > 2] } \\
\hline$\leq 1$ & 0,37 & $0,29-0,47$ & $<0,01$ \\
\hline \multicolumn{4}{|l|}{ Intervalo entre as visitas do enfermeiro (meses) [referência: > 2] } \\
\hline$>1 \mathrm{e} \leq 2$ & 0,35 & $0,29-0,42$ & $<0,01$ \\
\hline$\leq 1$ & 0,16 & $0,13-0,20$ & $<0,01$ \\
\hline \multicolumn{4}{|l|}{ Intervalo entre as visitas do fisioterapeuta (meses) [referência: > 2] } \\
\hline$>1 \mathrm{e} \leq 2$ & 0,70 & $0,50-0,98$ & $<0,01$ \\
\hline$\leq 1$ & 0,69 & $0,47-0,99$ & $<0,01$ \\
\hline Visita do nutricionista [referência: sem visita] & 0,66 & $0,50-0,86$ & $<0,01$ \\
\hline \multicolumn{4}{|c|}{ Grau de dependência para atividades básicas da vida diária [referência: independente] } \\
\hline Dependente total & 3,92 & $2,42-6,34$ & $<0,01$ \\
\hline Alimentação enteral [referência: oral] & 2,50 & $1,97-3,16$ & $<0,01$ \\
\hline \multicolumn{4}{|l|}{ Número de medicamentos em uso [referência: sem uso] } \\
\hline $1-5$ & 1,50 & $0,70-3,19$ & 0,30 \\
\hline Mais de 5 & 1,98 & $0,93-4,21$ & 0,08 \\
\hline \multicolumn{4}{|l|}{ Via de entrada de ar [referência: ar ambiente] } \\
\hline Oxigenioterapia & 1,64 & $1,24-2,17$ & $<0,01$ \\
\hline Traqueostomia & 1,56 & $0,72-3,38$ & 0,26 \\
\hline \multicolumn{4}{|l|}{ Úlcera de pressão [referência: pele íntegra] } \\
\hline Grau I ou II & 1,34 & $1,08-1,66$ & 0,01 \\
\hline Grau III ou IV & 1,76 & $1,15-2,69$ & 0,01 \\
\hline Internação no último trimestre [referência: sem internação] & 1,69 & $1,41-2,03$ & $<0,01$ \\
\hline \multicolumn{4}{|c|}{ Intervalo entre as consultas ambulatoriais com especialidades básicas (meses) [referência: > 2] } \\
\hline$\leq 1$ & 0,33 & $0,21-0,52$ & $<0,01$ \\
\hline
\end{tabular}

IC95\%: intervalo de 95\% de confiança.

modalidades de atenção domiciliar, como estratégias para ampliar as possibilidades de acesso do idoso ao cuidado, requerem avaliações locais de sua efetividade.

As principais causas de internação da população idosa observadas neste e em outros estudos 15,16 apontam para a possibilidade de prevenção se houver ambiente adequado e intervenção oportuna sobre seus fatores de risco, como é o caso das pneumonias 17 , fraturas de fêmur 18 e depleção de volume. O menor tempo livre de internação entre idosos de idade avançada, dependentes para atividades básicas da vida diária, cronicamente doentes e com uma internação hospitalar ou mais no trimestre anterior, é compatível com o observado em outros estudos 19,20. Ressalte-se que o custo, o tempo de permanência e as consequências deletérias da internação hospitalar costumam ser expres- sivamente maiores nos idosos frágeis, indicando a necessidade de intensificação dos cuidados hospitalares destes.

Minayo 21,22, ao analisar os desafios colocados para o setor saúde diante do vertiginoso crescimento da população idosa, que requer elevados recursos financeiros e cuidados humanos, destaca a baixa valorização das tecnologias leves em saúde, como os cuidados oferecidos por profissionais tecnicamente preparados. A utilização de outra tecnologia leve, como o aumento da cobertura das equipes de saúde da família do Sistema Único de Saúde (SUS), esteve associada à diminuição das internações por condições sensíveis à atenção primária dos idosos 23 . O presente estudo, ao demonstrar que o aumento da frequência de visitas domiciliares funcionou como fator protetor para internação nos pacientes do PrGC/AD, justifica mais investigações para esta- 
belecer o papel da atenção domiciliar na atenção ao idoso frágil.

É importante frisar que o equilíbrio entre o maior investimento em visitas domiciliares e a redução de custos decorrentes das internações é um assunto que deve ser abordado em estudos de custo-efetividade. Em análise de custo-efetividade conduzida na mesma coorte de pacientes (dados não publicados), observamos que, para aqueles de baixa complexidade, a frequência de visitas domiciliares em que é atingida a maior efetividade do PrGC/AD - medida pelo tempo livre de internação - é, também, custo-efetiva. Por outro lado, nos pacientes de alta complexidade, a frequência de visitas em que é atingida a máxima efetividade não é simultaneamente custo-efetiva. Em outras palavras, para se ter uma intervenção custo-efetiva neste grupo de pacientes, a oferta de frequência de visitas deve ser inferior ao que é necessário para se obter a maior efetividade. Essa relação reforça a necessidade de se avaliar criteriosamente o custo-efetividade das tecnologias em saúde.

Os resultados deste estudo devem ser vistos à luz das suas limitações. A principal delas refere-se à sua natureza observacional, o que não permitiu o controle de potenciais variáveis de confusão. Essa impossibilidade pode ter levado a associações espúrias entre o intervalo das visitas domiciliares dos profissionais de saúde e o tempo livre de internação hospitalar. Vieses comuns aos estudos observacionais, como o viés de indicação, também devem ser reconhecidos. Na presente pesquisa, viés de indicação pode ter acontecido caso a maior frequência de visitas de profissionais tenha sido diretamente influenciada pelo risco de internação avaliado pelos profissionais. No entanto, observe-se que este viés atuaria no sentido de reduzir o tamanho do efeito das visitas domiciliares no tempo livre de internação. Outra limitação do estudo refere-se à não disponibilidade de informações mais detalhadas da distribuição de doenças dos pacientes. $\mathrm{Na}$ ausência desses dados, o ajuste do risco de internação hospitalar no modelo de sobrevida de Cox baseou-se apenas nas variáveis clínicas disponíveis nas fichas de avaliação da auditoria (ver em Material e Métodos), o que pode ter resultado em ajuste insuficiente. Esse entrave reforça a necessidade de que as empresas de atenção domiciliar coletem sistematicamente dados precisos, completos e clinicamente relevantes, a fim de permitir avaliações válidas da efetividade do serviço oferecido aos pacientes.

A necessidade de se diferenciarem tecnologias sem benefícios para a saúde - ou com efeito deletério - das eficazes é ainda maior diante do surgimento de tecnologias geradoras de grandes dispêndios e das mudanças no perfil epidemiológico das populações 24 . Os resultados deste estudo possibilitam reflexões sobre a construção de indicadores de resultados dos programas de atenção domiciliar para idosos e sobre a análise de custo-efetividade desses programas. 


\section{Resumen}

Se realizó un estudio de cohorte retrospectivo para evaluar el impacto de un plan de asistencia del Programa de Atención Domiciliaria de Unimed-BH, modalidad de Gestión de Casos (PrGC/AD), sobre el tiempo libre de hospitalización en pacientes con 60 años o más. Se usó el modelo de riesgos proporcionales de Cox para evaluar el efecto del intervalo entre las visitas domiciliarias de los profesionales del programa sobre el tiempo libre de hospitalización de 2.943 ancianos, ajustado por edad, medicamentos usados, vía de alimentación, úlcera por presión, deterioro cognitivo, dependencia física, oxigenoterapia, consultas ambulatorias y hospitalizaciones en el trimestre anterior. Fueron factores de riesgo para un menor tiempo libre de hospitalización: grado de dependencia física, alimentación enteral, oxigenoterapia suplementaria, úlcera por presión y hospitalizaciones en el trimestre anterior. Las frecuencias de visitas médicas y de enfermeros tuvieron un efecto protector dosis-respuesta. Los resultados sugieren que las visitas domiciliarias regulares de médico y enfermero aumentan el tiempo libre de hospitalización en los pacientes asistidos por el $\mathrm{PrGC/AD}$.

Atención Domiciliaria de Salud; Anciano; Evaluación de Tecnologías de Salud

\section{Colaboradores}

F. M. Biscione, D. A. C. Szuster e E. F. Drumond participaram da concepção e desenho do projeto, da análise e interpretação dos dados e aprovaram a versão final do manuscrito. M. Turci colaborou na concepção do estudo, revisão da literatura e redação do manuscrito. G. U. A. Ferreira e J. F. Lima Júnior contribuíram na análise e interpretação dos dados e aprovaram a versão final do manuscrito. S. A. L. Bersan participou da concepção do projeto e da interpretação dos dados e aprovou a versão final do manuscrito.

\section{Agradecimentos}

Agradecemos ao Dr. Adebal de Andrade Filho, ao Dr. Luis Fernando Rolim Sampaio e à Dra. Ana Adalgisa de Oliveira Borges, responsáveis pelo serviço de atenção domiciliar da cooperativa. Agradecemos a todos os colaborados e funcionários da Unimed-BH que contribuíram para as diversas etapas deste estudo, em especial às enfermeiras Paula Cristina Ribeiro Nunes e Clarissa de Miranda Vieira.

\section{Conflito de interesses}

Todos os autores do artigo atuam como funcionários da Cooperativa de Trabalho Médico Unimed Belo Horizonte (Unimed-BH).

\section{Referências}

1. Giacomin KC, Peixoto SV, Uchoa E, Lima-Costa MF. Estudo de base populacional dos fatores associados à incapacidade funcional entre idosos na Região Metropolitana de Belo Horizonte, Minas Gerais, Brasil. Cad Saúde Pública 2008; 24:1260-70.

2. Lima-Costa MF, Firmo JOA, Uchoa E. The Bambuí Cohort Study of Aging: methodology and health profile of participants at baseline. Cad Saúde Pública 2011; 27 Suppl 3:S327-35.

3. Lima-Costa MF, Veras R. Saúde pública e envelhecimento. Cad Saúde Pública 2003; 19:700-1.

4. Lima-Costa MF, Matos DL, Camargos VP, Macinko J. Tendências em dez anos das condições de saúde de idosos brasileiros: evidências da Pesquisa Nacional por Amostra de Domicílios (1998, 2003, 2008). Ciênc Saúde Coletiva 2011; 16:3689-96.

5. Siqueira AB, Cordeiro RC, Perracini MR, Ramos LR. Impacto funcional da internação hospitalar de pacientes idosos. Rev Saúde Pública 2004; 38:687-94.
6. Veras R. Em busca de uma assistência adequada à saúde do idoso: revisão da literatura e aplicação de um instrumento de detecção precoce e de previsibilidade de agravos. Cad Saúde Pública 2003; 19:705-15.

7. Silva KL, Sena RR, Terenzi C, Feuerwerker LCM, Merhy EE. Atenção domiciliar como mudança do modelo tecnoassistencial. Rev Saúde Pública 2010; 44:166-76.

8. Valle EA, Bem Filho PM, Barroso ABP, Bersan SAL. Desenvolvimento de uma escala clínico-funcional para casos complexos. Rev Bras Geriatr Gerontol 2010; 13:533-7.

9. Katz S, Ford AB, Moskowitz RW, Jackson BA, Jaffe MW. Studies of illness in the aged. The index of ADL: a standardized measure of biological and psychosocial function. JAMA 1963; 185:94-9. 
10. Ministério da Saúde. Portaria no 2.528, de 19 de outubro de 2006. Aprova a Política Nacional de Saúde da Pessoa Idosa. Diário Oficial da União 2006; 20 out.

11. Veras RP. Envelhecimento populacional do Brasil: mudanças demográficas e desafios epidemiológicos. Rev Saúde Pública 1991; 25:476-88.

12. Floriani CA, Schramm FR. Atendimento domiciliar ao idoso: problema ou solução? Cad Saúde Pública 2004; 20:986-94.

13. van Haastregt J, Diederiks JPM, van Rossum E, de Witte LP, Crebolder H. Effects of preventive home visits to elderly people living in the community: systematic review. BMJ 2000; 320:745-58.

14. Elkan R, Kendrick D, Dewey M, Hewitt M, Robinson J, Blair M, et al. Effectiveness of home based support for older people: systematic review and metaanalysis. BMJ 2001; 323:719-25.

15. Dutra MM, Moriguchi EH, Lampert MA, Poli-de-Figueiredo CE. Validade preditiva de instrumento para identificação do idoso em risco de hospitalização. Rev Saúde Pública 2011; 45:106-12.

16. Loyola Filho AI, Matos DL, Giatti L, Afradique ME, Peixoto SV, Lima-Costa MF. Causas de internações hospitalares entre idosos brasileiros no âmbito do SUS. Epidemiol Serv Saúde 2004; 13:229-38.

17. Lima-Costa MF. Fatores associados à vacinação contra gripe em idosos na região metropolitana de Belo Horizonte. Rev Saúde Pública 2008; 42:100-7.

18. Siqueira FV, Facchini LA, Piccini RX, Tomasi E, Thumé E, Silveira DS, et al. Prevalência de quedas em idosos e fatores associados. Rev Saúde Pública 2007; 41:749-56.
19. Boult C, Downd B, McCaffrey D, Boult L, Hernandez R, Krulewitch H. Screening elders for risk of hospital admission. J Am Geriatr Soc 1993; 41: 811-7.

20. Macinko J, Camargos V, Firmo JOA, Lima-Costa MF. Predictors of 10-year hospital use in a community-dwelling population of Brazilian elderly: the Bambuí cohort study of aging. Cad Saúde Pública 2011; 27 Suppl 3:S336-44.

21. Minayo MCS. O envelhecimento da população brasileira e os desafios para o setor saúde. Cad Saúde Pública 2012; 28:208-10.

22. Minayo MCS. A ação humana como determinante para a efetividade dos tratamentos de saúde. Ciênc Saúde Coletiva 2011; 16:3030.

23. Macinko J, Oliveira VB, Turci MA, Guanais FC, Bonolo P, Lima-Costa MF. The Influence of hospital and primary care supply on ambulatory care sensitive hospitalizations among adults in Brazil 1999-2007. Am J Public Health 2011; 101:1963-70.

24. Departamento de Ciência e Tecnologia, Secretaria de Ciência e Tecnologia e Insumos Estratégicos, Ministério da Saúde. Avaliação de tecnologias em saúde: institucionalização das ações no Ministério da Saúde. Rev Saúde Pública 2006; 40:743-7.

Recebido em 31/Jan/2013

Versão final reapresentada em 28/Mai/2013

Aprovado em 12/Jul/2013 